\title{
Bioconvection of Micropolar Fluid in an Annulus
}

\author{
D. Srinivasacharya \\ Department of Mathematics, \\ National Institute of Technology, Warangal, \\ 506004, Telangana State, India. \\ Corresponding author: dsc@nitw.ac.in, dsrinivasacharya@yahoo.com \\ I. Sreenath \\ Department of Mathematics, \\ National Institute of Technology, Warangal, \\ 506004, Telangana State, India. \\ E-mail: sreenath.itikela@gmail.com
}

(Received December 16, 2018; Accepted September 16, 2019)

\begin{abstract}
This paper deals with the bioconvection of microploar fluid in an annulus containing microorganisms in which the outer cylinder is rotating. A mathematical model, with a fully coupled system of partial differential equations presenting the velocity, total mass, momentum, thermal energy, mass diffusion, and motile microorganisms is presented. A suitable transformations is adopted to reduce the governing non-linear governing to a set of non-linear ordinary differential equations and then linearized by means of successive linearization method. The resulign linearized equaions are solved using Chebyshev collocation method. The illustrating analysis of influences of the various flow governing physical parameters such as the micropolar coupling number, the bioconvection Schmidt-number, Prandtl number, Lewis number and bioconvection Peclet-number and Reynolds number on motile microorganism distribution are studied and is presented. Also, the density number of motile microorganism is examined for various governing parameters along with slip parameter of motile microorganism.
\end{abstract}

Keywords-Bioconvection, Micropolar fluid, Density number of motile microorganism.

\section{Introduction}

The fluid flow between an annulus region of two concentric cylinders attain significance attention of researchers due to its multi-ranged applications in industry and engineering. In particular, the analysis of heat as well as mass transfer in concentric cylinders, with the inner/outer (one or both) cylinder(s) rotate(s)/at stationary, is an important research area due to it's applicability in many industrial practices such as drilling, rotating machinery, swirl nozzles, ballistics of projectiles with spin, mechanical, chemical mixing equipments, electrical motors, transport of petroleum, food preparation, heat exchangers, cooling systems in electronic devices, electrical machineries with rotor and stator. Ever since (Couette et al., 1890) initiated the study of investigating viscous fluids flow patterns in the annulus region between co-rotating cylinders, a large number of hypothetical studies have been reported in the literature.

Several researchers have enticed in the study of the flow of non-Newtonian fluids, which exhibit a non-linear relationship between shear stress and shear rate, due to multifaceted range of applications in natural, industrial and technological flow processes. The fluid model formulated by (Eringen, 1966) called micropolar fluid, exhibit the effects of local rotary inertia along with couple stresses. This theory plays an instrumental role in explaining the flow of liquid crystal, colloidal fluids and animal blood, etc. The existence of dust particle or smoke particles in a gas, may be 
International Journal of Mathematical, Engineering and Management Sciences

Vol. 5, No. 2, 237-247, 2020

https://doi.org/10.33889/IJMEMS.2020.5.2.019

modeled with micropolar fluid dynamics. The mathematical theory and scientific aspects of this fluid theory are presented in the textbooks (Lukaszewicz, 1999; Eremeyev et al., 2012). Ariman et al. (1967) analyzed the flow of micropolar fluid between two concentric cylinders. Ramkissoon and Majumdar (1977) obtained exact solutions for the micropolar fluid flow through the annular region between concentric cylinders where the inner cylider is moving with time dependent angular velocity where as the outer cylinder is fixed. Agarwal and Dhanapal (1988) examined the micropolar fluid flow along with heat transfer through the annulus between coaxial porous cylinders when the outer cylinder moves parallel to itself at a constant velocity. Mahmud and Fraser (2002) analysed the second law of micropolar flow through concentric cylinders. Srinivasacharya and Shiferaw (2013) consideredSoret and Dufour effects on micropolar fluid flow in annulus region with first-order chemical reaction combined with magnetic field. Srinivasacharya and Bindu (2016) investigated the influence of convective boundary conditions and the velocity slip on the entropy generation in a micropolar fluid flow through porous annulus.

Bioconvection is due to microscopic convection generated by the density gradient produced by the collective motion of self-propelled motile microorganisms within the fluid. Bioconvection has many applications in biological systems and biotechnology. Microorganisms are hugely suspended in aqueous environments, such as droplets, puddles, rivers even in oceans. Childress et al. (1975) were the firs to develop the theory of gravitactic bioconvection for geotactic microorganisms. Later, Pedley et al. (1988) developed a continuum model for a suspension of swimming gyrotactic microorganisms. Alloui et al. (2005) solved numerically the Navier - Stokes equation coupled with the microorganism conservation equation describing the gravitactic microorganism in a vertical cylinder using control volume method. Taheri and Bilgen (2008) investigated the progress of bioconvection in a stress free sidewalls vertical cylinder with the influence of cooling or heating from below at uniform temperature. Raees et al. (2015) considered the unsteady mixed nano bioconvection flow in a horizontal channel. Mosayebidorcheh et al. (2017) analyzed the bioconvection flow in a horizontal channel filled with nanofluid which contains both nanoparticles and gyrotactic microorganisms.

The literature review reveals that no study exists on the behaviour of microorganisms in a micropolar fluid flow in an annulus between concentric cylinders. This study investigates the bioconvection in an annulus with micropolar fluid in which the outer cylinder is rotating with the angular velocity.

\section{Mathematical Formulation}

Consider the steady flow of an incompressible micropolar fluid containing motile microorganisms between two concentric cylinders. As depicted in the Figure 1, a cylindrical polar coordinate system $(r, \varphi, z)$ is taken with $\mathrm{z}$-axis as the common axis for both cylinders. Assume that the outer cylinder rotates with constant angular velocity $\Omega$ while the inner is at rest. The rotation of the outer cylinder generates the flow in the annulus region. Let the radii of the inner and outer cylinders are $a$ and $b$ respectively. The inner and outer cylinders are maintained at the constant temperatures $T_{a}$ and $T_{b}$, constant concentrations $C_{a}$ and $C_{b}$ and constant concentration of motile microorganisms $\vartheta_{a}$ and $\vartheta_{b}$. Hence, the equations governing present flow are given by: 


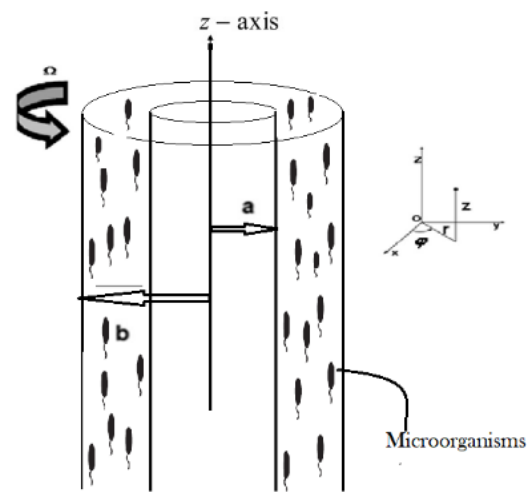

Figure 1. Geometry of the problem

$$
\begin{aligned}
& \frac{\partial u}{\partial \varphi}=0 \\
& \frac{\partial P}{\partial r}=\frac{\rho u^{2}}{r} \\
& \left(\frac{\mu+\kappa}{\rho}\right)\left(\frac{\partial^{2} u}{\partial r^{2}}+\frac{1}{r} \frac{\partial u}{\partial r}+\frac{\partial^{2} u}{\partial z^{2}}-\frac{u}{r^{2}}\right)-\frac{\kappa}{\rho} \frac{\partial \varpi}{\partial r}=0 \\
& \frac{\kappa}{\mu}\left(\frac{\partial u}{\partial r}+\frac{u}{r}\right)+\frac{\gamma}{\rho}\left(\frac{1}{r} \frac{\partial \varpi}{\partial r}+\frac{\partial^{2} \varpi}{\partial r^{2}}\right)-\frac{2 \kappa}{\rho} \varpi=0 \\
& \alpha\left(\frac{\partial^{2} T}{\partial r^{2}}+\frac{1}{r} \frac{\partial T}{\partial r}\right)-2 k\left(\frac{1}{2 r} \frac{\partial(r \mathrm{u})}{\partial r}-\varpi\right)^{2}+(\mu+k)\left(\frac{\partial u}{\partial r}-\frac{u}{r}\right)^{2}+\gamma\left(\frac{\partial \varpi}{\partial r}\right)^{2} \\
& D_{B}\left(\frac{\partial^{2} C}{\partial r^{2}}+\frac{1}{r} \frac{\partial C}{\partial r}\right)-k_{1}\left(\mathrm{C}-\mathrm{C}_{a}\right)=0 \\
& \frac{b_{c} w_{c}}{C_{1}-C_{2}} \frac{\partial}{\partial r}\left(\vartheta \frac{\partial C}{\partial r}\right)=D_{n}\left(\frac{\partial^{2} \vartheta}{\partial r^{2}}+\frac{1}{r} \frac{\partial \vartheta}{\partial r}\right)
\end{aligned}
$$

with the following boundary conditions:

$$
\begin{aligned}
& u=0 \quad \varpi=0 \quad T=T_{a} \quad C=\mathrm{C}_{a} \quad \vartheta=\vartheta_{a} \quad \text { at } \quad r=a, \\
& u=b \Omega \quad \varpi=\frac{1}{2 r} \frac{\partial}{\partial r}(r a) \quad T=T_{b} \quad C=\mathrm{C}_{b} \quad \vartheta=\vartheta_{b} \quad \text { at } \quad r=b,
\end{aligned}
$$

where $u$ is the velocity components along the $\varphi$ direction, $\varpi$ is the azimuthal component of microrotation vector, $T$ is the temperature, $C$ is the concentration, $\vartheta$ is the density of the motile microorganism, $\rho$ is the density, $\mu$ is the dynamic viscosity, $\kappa$ and $\gamma$ are the microrotation and spin gradient viscosities, respectively, $\alpha$ is the thermal diffusivity, $D_{b}$ is the mass diffusivity, $k_{l}$ is the chemical reaction parameter, $D_{n}$ is the micro-organism diffusivity constant, $b_{c}$ is the Chemotaxis 
International Journal of Mathematical, Engineering and Management Sciences

Vol. 5, No. 2, 237-247, 2020

https://doi.org/10.33889/IJMEMS.2020.5.2.019

constant and $w_{C}$ is the maximum cell swimming speed.

Substituting the following similarity transformation variables

$$
\begin{aligned}
& r^{2}=b^{2} \eta, \quad u=\left(\frac{\Omega}{\sqrt{\eta}}\right) f(\eta), \quad \varpi=\left(\frac{\Omega}{b}\right) \mathrm{h}(\eta), \quad T=T_{0}+\left(\mathrm{T}_{a}-\mathrm{T}_{b}\right) \theta(\eta), \\
& C=C_{0}+\left(\mathrm{C}_{a}-C_{b}\right) \phi(\eta), \quad \vartheta=\vartheta_{a} \chi(\eta),
\end{aligned}
$$

Into equation (3) - (7), we get

$$
\begin{aligned}
& f^{\prime \prime}-N h^{\prime}=0 \\
& 2 G\left(h^{\prime}+\eta h^{\prime \prime}\right)-h+f^{\prime}=0 \\
& \eta^{3} \theta^{\prime \prime}+\eta^{2} \theta^{\prime}+\frac{B r}{1-N}\left[\frac{N}{2} \eta^{2}\left(f^{\prime}-h\right)^{2}+\left(\eta f^{\prime}-\mathrm{f}\right)^{2}+\frac{G}{2} N \eta^{3} g^{\prime 2}\right]=0 \\
& \frac{1}{L e \cdot P r} \eta \phi^{\prime \prime}+\frac{1}{L e \cdot P r} \phi^{\prime}-\frac{K}{4} \phi=0 \\
& \frac{1}{S c}\left(\eta \chi^{\prime \prime}+\chi^{\prime}\right)-\frac{P e}{S c}\left(\eta\left(\chi^{\prime} \phi^{\prime}+\chi \phi^{\prime \prime}\right)+\frac{1}{2} \chi \phi^{\prime}\right)=0
\end{aligned}
$$

The associted boundary conditions are

$$
\begin{aligned}
& f\left(\eta_{0}\right)=0, \quad \mathrm{~h}\left(\eta_{0}\right)=0, \quad \theta\left(\eta_{0}\right)=0, \quad \phi\left(\eta_{0}\right)=1, \quad \chi\left(\eta_{0}\right)=1 \\
& f(1)=0, \quad \mathrm{~h}(1)=\left(\frac{d f}{d \eta}\right)_{\eta=1}, \quad \theta(1)=\delta \theta, \quad \phi(1)=\delta \phi, \quad \chi(1)=\delta \chi
\end{aligned}
$$

where the prime denotes derivative with respect to $\eta$, and $\eta_{0}=\left(\frac{a}{b}\right)^{2}$ which correspons to the inner cylinder radius, $N=\frac{\kappa}{\mu+\kappa}$ is the coupling number, $R e=\frac{\Omega b \rho}{\mu}$ is a Reynolds number, $m^{2}=\frac{b^{2} \kappa(2 \mu+\kappa)}{\gamma(\mu+\kappa)}$ is the micropolar parameter, $\operatorname{Pr}=\frac{v}{\alpha}$ is the Prandtl number, $L e=\frac{\alpha}{D_{B}}$ is the Lewis number, $S c=\frac{v}{D_{n}}$ is the bioconvection Schmidt number, $P e=\frac{b_{c} m_{c}}{D_{n}}$ is the bioconvection Peclet number and $\delta_{\theta}=$ $\frac{T_{b}-T_{0}}{T_{a}-T_{0}}, \delta_{\phi}=\frac{C_{b}-C_{0}}{C_{a}-C_{0}}$ and $\delta_{\chi}=\frac{\vartheta_{b}}{\vartheta_{a}}$ are constants.

The physical quantities of practical interest, which describe the flow characteristics about microorganism transfer rate is the the local wall motile microorganism flux $Q_{x}=$ $\frac{x}{k\left(\vartheta_{b}-\vartheta_{a}\right)}\left[\frac{\partial \vartheta}{\partial \eta}\right]_{r=a a n d r=b}$ are calculated. The non-dimenaional form of this quantities is given by $R e^{-\frac{1}{2}} Q_{x}=-\chi^{\prime}(\eta)$ at $\eta=\eta_{0}$ and $\eta=1$. 
International Journal of Mathematical, Engineering and Management Sciences

Vol. 5, No. 2, 237-247, 2020

https://doi.org/10.33889/IJMEMS.2020.5.2.019

\section{Method of Solution}

The system of nonlinear coupled differential equations (10) - (14) are reduced to a system of linear ordinary differential equations using the successive linearizion method (SLM) (Motsa and Shateyi, 2011). The resulting system of linearized ordinary differential equations is solved by Chebyshev spectral collocation method.

Let, $\mathbb{F}(\eta)=[f(\eta), h(\eta), \theta(\eta), \phi(\eta), \chi(\eta)]$ and assumed that it can be expressed as

$\mathbb{F}(\eta)=\mathbb{F}_{\tilde{\imath}}(\eta)+\sum_{k=0}^{\tilde{\imath}-1} \mathbb{F}_{k}(\eta), \quad \tilde{\imath}=1,2,3, \ldots$

where $\mathbb{F}_{\tilde{\imath}}(\eta)$ are unknown functions and $\mathbb{F}_{k}(\eta),(0 \leq k \leq(\tilde{\imath}-1))$ are approximations from the previous iterations. Substituting Eq.(16) in the governing equations (10) to (14) and retaining the linear terms of $\mathbb{F}_{\tilde{\imath}}(\eta)$, we obtain the following the system of ordinary differential equations.

$$
\begin{aligned}
& {[2 /(1-\mathrm{N})] f_{\tilde{i}}^{\prime \prime}-[N /(1-\mathrm{N})] h_{\tilde{i}}^{\prime \prime}=r_{1, \tilde{i}-1}^{*}} \\
& f_{\tilde{i}}^{\prime}+a_{1, \tilde{i}-1} h_{\tilde{i}}^{\prime \prime}+2 G h_{i}^{\prime}-h_{\tilde{i}}=r_{2, \tilde{i}-1}^{*} \\
& b_{1, \tilde{i}-1} f_{\tilde{i}}{ }^{\prime}+b_{2, \tilde{i}-1} f+b_{3, \tilde{i}-1} h_{\tilde{i}}{ }^{\prime}+b_{4, \tilde{i}-1} h_{\tilde{i}}+b_{5, \tilde{i}-1} \theta_{\tilde{i}}{ }^{\prime \prime}+b_{6, \tilde{i}-1} \theta_{\tilde{i}}{ }^{\prime}=r_{3, \tilde{i}-1}^{*} \\
& c_{1, \tilde{i}-1} \phi_{i}^{\prime \prime}+c_{2, \tilde{i}-1} \phi_{i}^{\prime}+c_{3, \tilde{i}-1} \phi_{\tilde{i}}^{\prime}=r_{4, \tilde{i}-1}^{*} \\
& d_{1, \tilde{i}-1} \phi_{\tilde{i}}^{\prime \prime}+d_{2, \tilde{i}-1} \phi_{r}{ }^{\prime}+d_{3, \tilde{i}-1} \chi_{\tilde{i}}^{\prime \prime}+d_{4, \tilde{i}-1} \chi_{\tilde{i}}{ }^{\prime}+d_{5, \tilde{i}-1} \chi_{\tilde{i}}=r_{5, \tilde{i}-1}^{*}
\end{aligned}
$$

where, the coefficients $a_{1, \tilde{\imath}-1}, b_{l, \tilde{\imath}-1}, c_{l, \tilde{\imath}-1}, l=1,2,3$ and $d_{k, \tilde{l}-1}, k=1,2, \ldots 6, r_{s, \tilde{l}-1}^{*}, s=1,2, \ldots 5$ are in terms of the approximations $\mathbb{F}_{k}(\eta),(0 \leq k \leq(\tilde{l}-1))$ and their derivatives.

The respective boundary conditions associated with the above equations are

$$
\begin{aligned}
& f_{i}\left(\eta_{0}\right)=0, \quad \mathrm{~h}_{i}\left(\eta_{0}\right)=0, \quad \theta_{i}\left(\eta_{0}\right)=0, \quad \phi_{i}\left(\eta_{0}\right)=1, \quad \chi_{i}\left(\eta_{0}\right)=1 \\
& f_{i}(\eta=1)=0, \quad \mathrm{~h}_{i}(\eta=1)=\left(\frac{d f}{d \eta}\right)_{\eta=1}, \theta_{i}(\eta=1)=\delta \theta, \quad \phi_{i}(\eta=1)=\delta \phi, \quad \chi_{i}(\eta=1)=\delta \chi
\end{aligned}
$$

By taking the initial approximation $\mathbb{F}_{0}(\eta)$, such that they satisfy boundary conditions (15), solving the system of equations (17) to (21) recursively, we get the approximate solutions for $\mathbb{F}(\eta)$,

Now, we solve the linearized equations (17) to (21) by Chebyshev collocation method (Canuto et al., 2006). To implement it, first the domain $\left[\eta_{0}, 1\right]$ is converted to $[-1,1]$ by the conversion $\eta=$ $\frac{\left(1-\eta_{0}\right) \tau_{m}+\left(1+\eta_{0}\right)}{2},-1 \leq \tau_{m} \leq 1$. In this procedure, the functions $\mathbb{F}_{r}(\eta)$ approximated by the Chebyshev polynomials and these polynomials are collocated at $J+1$ Gauss-Lobatto points in the interval $[-1,1]$.

Expressing the unknown functions $\mathbb{F}_{i}(\eta)$ and its derivative at the Gauss-Lobatto collocation points 
International Journal of Mathematical, Engineering and Management Sciences

Vol. 5, No. 2, 237-247, 2020

https://doi.org/10.33889/IJMEMS.2020.5.2.019

and then substituting them into the equations (17)-(22) leads to a system of algebraic equations. Writing the boundary conditions in terms of Chebyshev polynomials at the collocation points, incorporating them in the system and then solving the resulting matrix system, we get the solution.

\section{Results and Discussion}

The effect of various non-dimensional physical parameters such as the coupling number $N$, micropolar parameter $m$, the Raynald number $R e$, the Prandtl number $P r$, the bioconvection Lewis number $L e$, the bioconvection Schmidt number $S c$ and the bioconvection Peclet number $P e$ on the non-dimensional density of motile microorganisms are presented graphically in Figures 2- 6. An extensive computations have been carried out by assigning default values as $N=0.5, \mathrm{Le}=1$, Sc $=0.22, \mathrm{Pe}=1, \operatorname{Pr}=0.71$ and $\mathrm{G}=4, \mathrm{Br}=0.1, \mathrm{~K}=5$ along with the slip parameter such as $\delta \phi=0$, $\delta \chi=1, \delta \theta=0.2$ which are not against the physical requirements.

\subsection{Effect of Chemical Reaction Parameter K, Lewis Number Le, and Prandtl Number $\operatorname{Pr}$ on $\chi$}

The variation of motile microorganism by various parameters such as $\mathrm{K}, L e$ and $\operatorname{Pr}$ is visualized in Figure 2. It is depicted from Figure (2a) that the motile microorganism increases as the chemical reaction parameter increase i.e., the density of motile microorganism is directly proportional to the $\mathrm{K}$. For a given $\mathrm{K}$ value, the motile microorganism is decreasing near the innner cylinder and increasing towards the rotating outer cylinder. The reason can be understood that as the outer cylinder rotates the motile microorganisms are assumed to gather at the outer cylinder which causes an increse in the densty of the motile microorganism. From Figures (2b)-(2c), it is percieved that the motile microorganisms is rising with the rise in the values of $L e$ and $\operatorname{Pr}$. For a particular values of $L e$ and $\mathrm{Pr}$, the motile microorganism is reducing initially near the inner cylinder and then raising towards the outer cylinder.

The variation of motile microorganism for varying bioconvection parameters $P e$ is poltted in Figure (2d). For a fixed $P e$, the motile microorganism is reducing initially near the inner cylinder till the mid of the annulus region and then rising towards the outer cylinder. It is seen that $P e$ increases the density of motile microorganism decreases. 


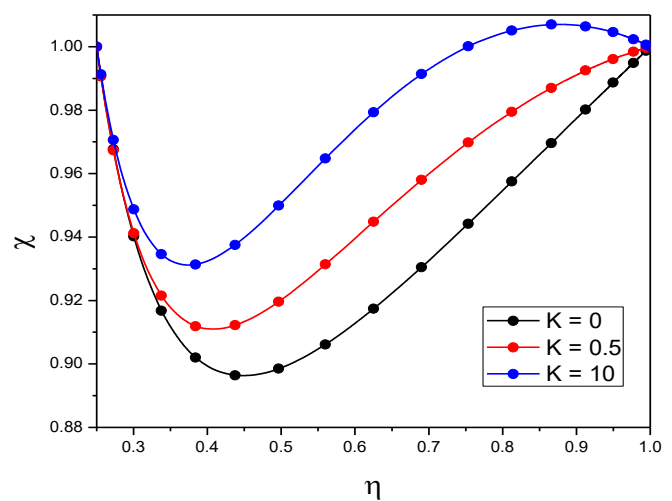

(a)

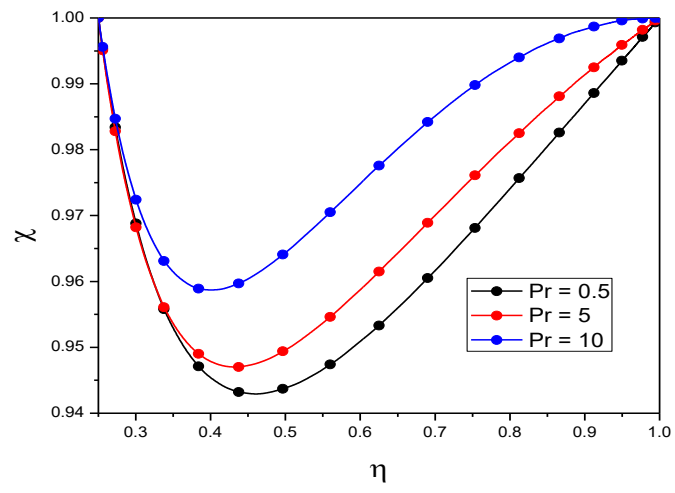

(c)

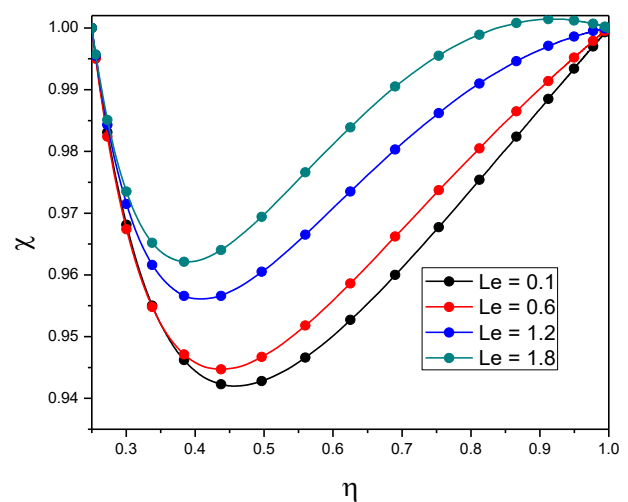

(b)

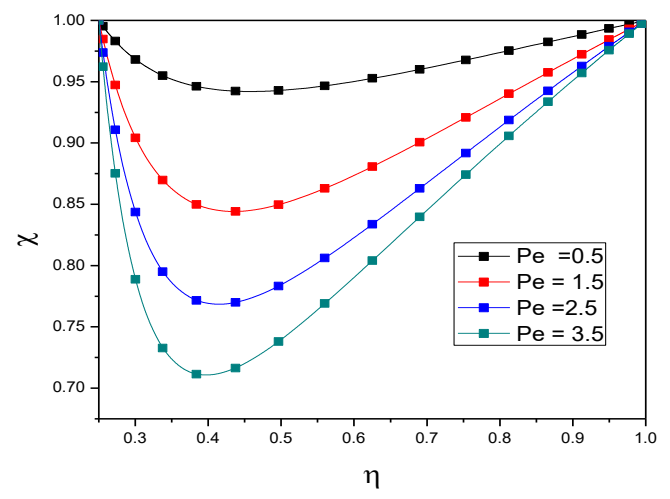

(d)

Figure 2. Profiles of $\chi$ w.r.t the parameter chemical reaction parameter $(K)$, Lewis number $(L e)$, Prandtl number $(P r)$, bioconvection Peclet number $(P e)$

\subsection{Effect of Slip Parameters $\delta \phi$ and $\delta \chi$ on $\chi$}

The influence of concentration slip parameter $\delta \phi$ and the motile microorganism slip parameter $\delta \chi$ on the motile microorganisms is exhibited in the Figure 3. The motile microorganisms, as illustrated in Figure (3a), is inversely proportional to $\delta \phi$. For a given $\delta \phi$, a notable impact on the behavior of motile microorganisms $\chi(\eta)$ is observed i.e., the density of motile microorganisms is reducing near the inner cylinder and increasing towards outer cylinder. As $\delta \chi$ increases the motile microorganism is also increasing, as depicted in Figure (3b), It is clear that the density motile microorganism decreases for a given value of the slip parameter value $\delta \chi$ as shown Figure (3b). 


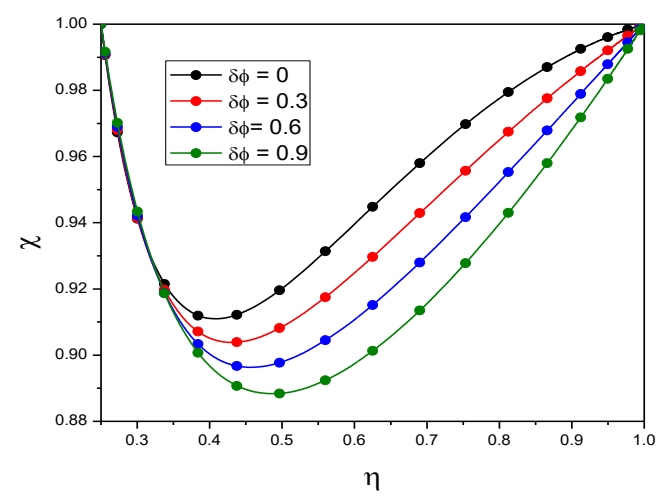

(a)

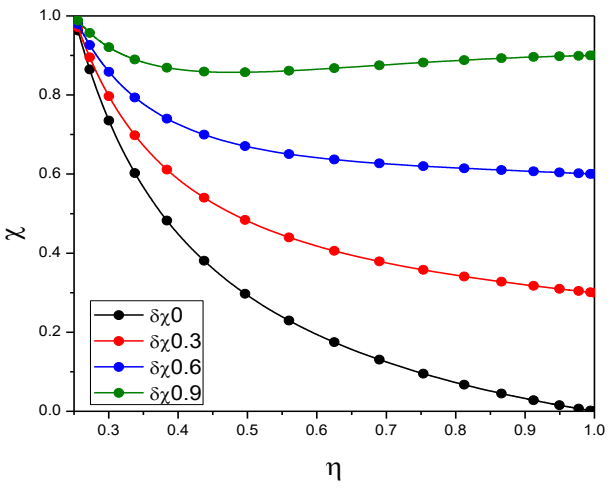

(b)

Figure 3. Profiles of $\chi$ w.r.t. the slip parameters in concentration and motile-microorganism $\delta \phi, \delta \chi$

\subsection{Effect of Flow Governing Parameters K, $L e$, and $\operatorname{Pr}$ on Density Number of Motile Microorganism $\boldsymbol{Q}_{\boldsymbol{x}}$}

The variation in density number of motile microorganism $\left(Q_{x}\right)$ with the parameters $\mathrm{K}, L e$ and $P r$ and $P e$ is depicted in Figure (4). The $Q_{x}$ at the inner cylinder is exhibited in Figures (4a), (4c), (4e) and $(4 \mathrm{~g})$ and for the outer cylinder in Figures $(4 \mathrm{~b}),(4 \mathrm{~d}),(4 \mathrm{f})$ and $(4 \mathrm{~h})$ respectively. At the inner cylinder, $Q_{x}$ is increasing function of $\mathrm{K}$. It shows a decreasing pattern for the given value of chemical reaction parameter $\mathrm{K}$ at the both the cylinders with an increase in motile microorganism slip parameter. A similar trend is observed for fixed values of $L e$ at the inner and outer cylinders. As the motile microorganism slip parameter increases, $Q_{x}$ is decreasing at the inner cylinder and increasing at the outer cylinder for fixed value of $P r$. For a fixed value of $P e$, see Figure $(4 \mathrm{~g})$ the density number of motile microorganism increases at the inner cylinder and the pattern is completely reversed i.e. the density number of motile microorganism decreases with an increases in $\mathrm{Pe}$ as shown in the Figure (4h). For a given $P e$, the density number of motile microorganism decreases at the inner and outer cylinders i.e., the density number of motile microorganism is inversely proportional to the motile microorganism slip parameter at the inner and outer cylinders. 
International Journal of Mathematical, Engineering and Management Sciences

Vol. 5, No. 2, 237-247, 2020

https://doi.org/10.33889/IJMEMS.2020.5.2.019

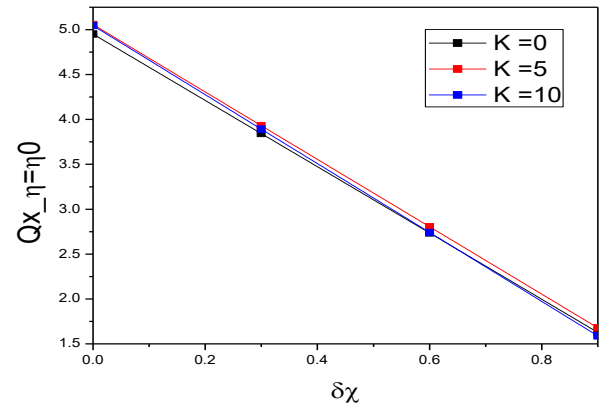

(a)

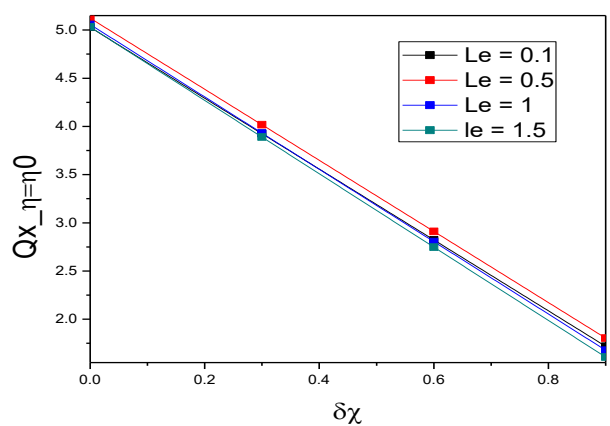

(c)

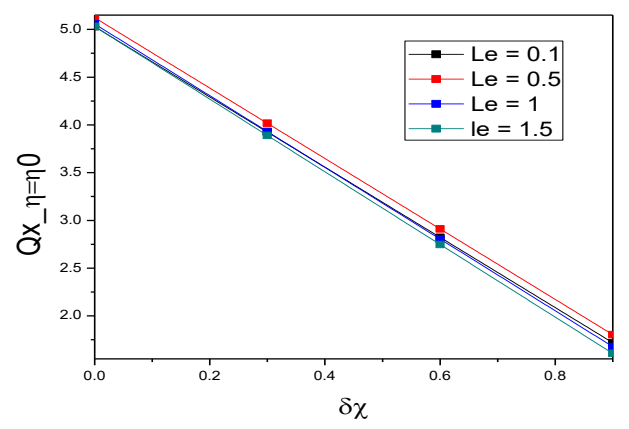

(e)

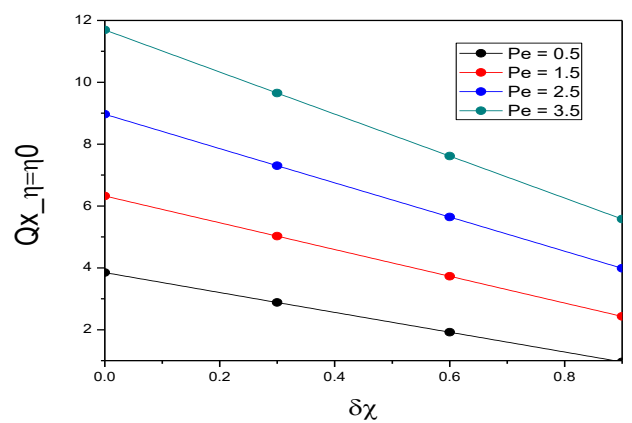

(g)

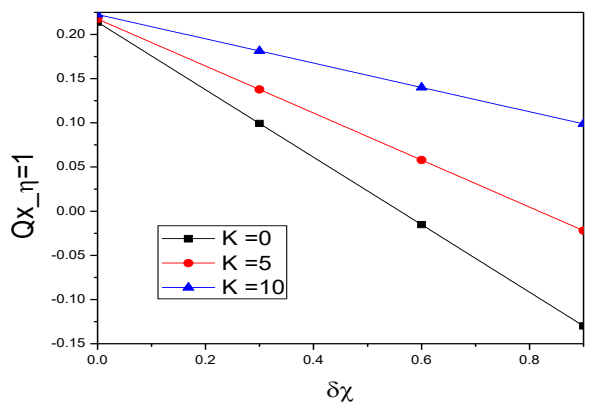

(b)

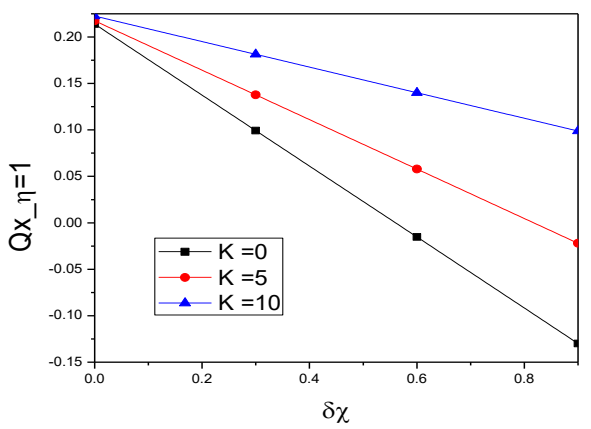

(d)

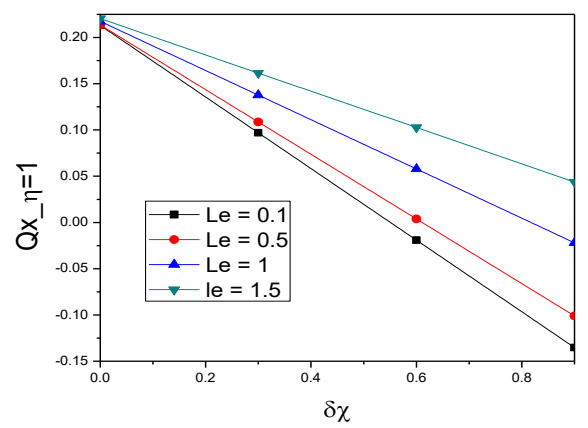

(f)

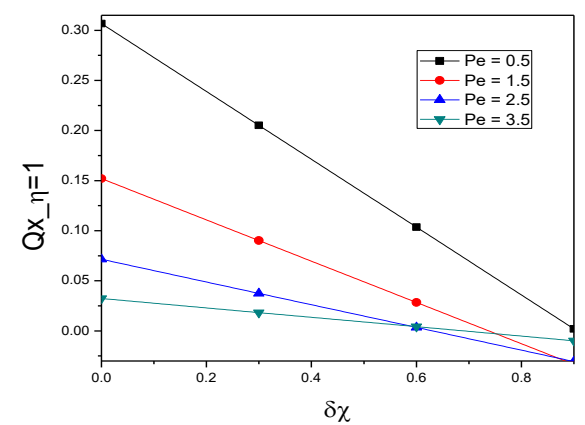

(h)

Figure 4. Profiles of profiles of $Q_{x}$ with $\delta \chi$ w.r.t K, $L e, \operatorname{Pr}$ and $P e$ at the bounadries of inner and outer cylinders 
International Journal of Mathematical, Engineering and Management Sciences

Vol. 5, No. 2, 237-247, 2020

https://doi.org/10.33889/IJMEMS.2020.5.2.019

\section{Conclusions}

The present study gives numerical solutions for the flow of a incompressible micropolar fluid containing microorganisms between two concentric cylinders with the rotating outer cylinder.

- The dimensionless density of motile microorganism $(\chi)$ increases as the chemical parameter $\mathrm{K}$, $\mathrm{Pr}$, and Le increases. And, inversely proportinal to the bioconvection parameter Pe. The slip parameters $(\delta \phi)$ shows decreaseing pattern and reversed scenario is observed for other parameter of slip in microorganism $\delta \chi$.

- The density number of motile microorganism $\left(Q_{x}\right)$ shows increasing pattern at the inner cylinder and outer cylinder wirh respect to the parameters $\mathrm{K}, L e, P r$, except for the bioconvection parameter $P e$ which is decreasing pattern at the outer cylinder.

\section{Conflict of Interest}

Both the authors have equal contribution in this work and it is declared that there is no conflict of interest for this publication.

\section{Acknowledgment}

The authors express their sincere gratitude to the referees for their valuable suggestions towards the improvement of the paper.

\section{References}

Agarwal, R.S., \& Dhanapal, C. (1988). Numerical solution of micropolar fluid flow and heat transfer between two co-axial porous circular cylinders. International Journal of Engineering Science, 26(11), 11331142 .

Alloui, Z., Nguyen, T.H., \& Bilgen, E. (2005). Bioconvection of gravitactic microorganisms in a vertical cylinder. International Communications in Heat and Mass Transfer, 32(6), 739-747.

Ariman, T., Cakmak, A.S., \& Hill, L.R. (1967). Flow of micropolar fluids between two concentric cylinders. The Physics of Fluids, 10(12), 2545-2550.

Canuto, C., Hussaini, M.Y., Quarteroni, A., \& Zang, T.A. (2006). Spectral methods: fundamentals in single domains. Scientific Computation Series. Berlin Heidelberg, Springer-Veralg.

Childress, S., Levandowsky, M., \& Spiegel, E.A. (1975). Pattern formation in a suspension of swimming microorganisms: equations and stability theory. Journal of Fluid Mechanics, 69(3), 591-613.

Couette, M. (1890). Studies relating to the friction of liquids. Annales de Chimie et de Physique, 21(6), 433510.

Eremeyev, V.A., Lebedev, L.P., \& Altenbach, H. (2012). Foundations of micropolar mechanics. Springer, Verlag, Berlin, Heidelberg.

Eringen, A.C. (1966). Theory of micropolar fluids. Journal of Mathematics and Mechanics, 16(1), 1-18.

Lukaszewicz, G. (1999). Micropolar fluids: theory and applications. Birkhauser, Boston, MA, USA.

Mahmud, S., \& Fraser, R.A. (2002). Second law analysis of heat transfer and fluid flow inside a cylindrical annular space. Exergy-An International Journal, 2(4), 322-329. 
International Journal of Mathematical, Engineering and Management Sciences

Vol. 5, No. 2, 237-247, 2020

https://doi.org/10.33889/IJMEMS.2020.5.2.019

Mosayebidorcheh, S., Tahavori, M.A., Mosayebidorcheh, T., \& Ganji, D.D. (2017). Analysis of nanobioconvection flow containing both nanoparticles and gyrotactic microorganisms in a horizontal channel using modified least square method (MLSM). Journal of Molecular Liquids, 227, 356-365.

Motsa, S., \& Shateyi, S. (2011). Successive Linearisation Solutiony of Free Convection Non-Darcy Flow with Heat and Mass Transfer. Advanced Topics in Mass Transfer, 19, 425-438.

Pedley, T.J., Hill, N.A., \& Kessler, J.O. (1988). The growth of bioconvection patterns in a uniform suspension of gyrotactic micro-organisms. Journal of Fluid Mechanics, 195, 223-237.

Raees, A., Xu, H., \& Liao, S.J. (2015). Unsteady mixed nano-bioconvection flow in a horizontal channel with its upper plate expanding or contracting. International Journal of Heat and Mass Transfer, 86, 174182.

Ramkissoon, H., \& Majumdar, S.R. (1977). Unsteady flow of a micro-polar fluid between two concentric circular cylinders. The Canadian Journal of Chemical Engineering, 55(4), 408-413.

Srinivasacharya, D., \& Bindu, K.H. (2016). Entropy generation in a porous annulus due to micropolar fluid flow with slip and convective boundary conditions. Energy, 111, 165-177.

Srinivasacharya, D., \& Shiferaw, M. (2013). Cross diffusion effects on chemically reacting magnetohydrodynamic micropolar fluid between concentric cylinders. Journal of Heat Transfer, 135(12), 122003.

Taheri, M., \& Bilgen, E. (2008). Thermo-bioconvection in a suspension of gravitactic micro-organisms in vertical cylinders. International Journal of Heat and Mass Transfer, 51(13-14), 3535-3547. 